\title{
Reactive Oxygen Species in Pathogen Clearance: The Killing Mechanisms, the Adaption Response, and the Side Effects
}

\section{OPEN ACCESS}

Edited by: Kirkwood M. Land

University of the Pacific, United States

Reviewed by:

Tim Maisch,

University of Regensburg, Germany Kristin M. Burkholder, University of New England,

United States

*Correspondence:

Lei Cheng

chenglei@scu.edu.cn

Biao Ren

renbiao@scu.edu.cn

Specialty section:

This article was submitted to

Antimicrobials, Resistance

and Chemotherapy,

a section of the journal

Frontiers in Microbiology

Received: 28 October 2020

Accepted: 28 December 2020

Published: 04 February 2021

Citation:

Li H, Zhou X, Huang Y, Liao B, Cheng $L$ and Ren $B$ (2021) Reactive

Oxygen Species in Pathogen Clearance: The Killing Mechanisms, the Adaption Response, and the Side Effects. Front. Microbiol. 11:622534.

doi: 10.3389/fmicb.2020.622534
Hao Li, Xuedong Zhou, Yuyao Huang, Binyou Liao, Lei Cheng* and Biao Ren*

State Key Laboratory of Oral Diseases, National Clinical Research Center for Oral Diseases, West China Hospital of Stomatology, Sichuan University, Chengdu, China

Reactive oxygen species (ROS) are attractive weapons in both antibiotic-mediated killing and host-mediated killing. However, the involvement of ROS in antibiotic-mediated killing and complexities in host environments challenge the paradigm. In the case of bacterial pathogens, the examples of some certain pathogens thriving under ROS conditions prompt us to focus on the adaption mechanism that pathogens evolve to cope with ROS. Based on these, we here summarized the mechanisms of ROS-mediated killing of either antibiotics or the host, the examples of bacterial adaption that successful pathogens evolved to defend or thrive under ROS conditions, and the potential side effects of ROS in pathogen clearance. A brief section for new antibacterial strategies centered around ROS was also addressed.

Keywords: reactive oxygen species, secondary damage, metabolism remodeling, virulence, antibiotic resistance, antibiotic tolerance

\section{INTRODUCTION}

Reactive oxygen species (ROS), including hydrogen peroxide $\left(\mathrm{H}_{2} \mathrm{O}_{2}\right)$, hydroxyl radical (OH-), singlet oxygen $\left({ }^{1} \mathrm{O}_{2}\right)$, and superoxide anion $\left(\cdot \mathrm{O}_{2}{ }^{-}\right)$, are produced by the pathogen itself (endogenous) as a byproduct of aerobic respiration; they can also be encountered in the host environment (exogenous). ROS have been called "double-edged swords of life" (Mittler, 2017) in pathogen clearance. First, since ROS can directly damage DNA, lipids, and proteins, they are thought to be the weapon used by both antibiotics and the host immune system. However, controversies challenge the paradigm. Second, successful pathogens exploit ROS for their own adaption. This minireview aims to summarize the mechanisms of ROS-mediated killing by either antibiotics or the host, examples of pathogens that cope with the ROS conditions, the potential side effects of ROS in pathogen clearance, and novel antibacterial strategies centered around ROS. 


\section{ENDOGENOUS ROS, AS SECONDARY DAMAGE, FORMED IN RESPONSE TO ANTIBIOTIC EXPOSURE}

In general, antibiotics are thought to kill microbes through interaction with specific intracellular targets (Kohanski et al., 2010b). However, in 2007, Collins group proposed a novel mechanism of quinolones-induced killing by the generation of endogenous hydroxyl radicals (Dwyer et al., 2007). Since then, ROS have been shown to play a central role in lethality of numerous classes of bactericidal antibiotics, regardless of their specific targets (Kohanski et al., 2007). However, the role of ROS in antibiotic lethality became controversial and has been challenged (Keren et al., 2013; Imlay, 2015), since antibiotics can kill in the absence of ROS. The paradox contained in these statements can be solved by the idea that killing can derive from the primary damage of antibiotic or from a secondary, lethal stress response mediated by ROS (Zhao and Drlica, 2014; Zhao et al., 2015; Luan et al., 2018). If primary damage with specific targets is severe enough, it can result in death directly. Otherwise, primary damage stimulates a pathway that leads to ROS accumulation as secondary damage. New evidence for this hypothesis was provided in Escherichia coli. Hong et al. (2019) described a novel experimental system in which the role of secondary ROS damage could be tested in isolation. In this case, even after complete removal of quinolones, ROS accumulation and cell death continued to occur, indicating that when secondary ROS damage exceeds a critical threshold, it becomes a self-amplifying process and the terminal stage when bacteria responds to antibiotics.

However, how primary damage leads to ROS accumulation remained unclear. Since target-specific damage occurs in antibiotic killing, pathways leading to ROS accumulation most probably have drug-specific context as well. While different studies suggest that primary drug-target damage may activate processes such as envelopes stress response and programmed cell death, leading to ROS accumulation (Lobritz et al., 2015; Van Acker and Coenye, 2017; Stokes et al., 2019), most evidence is provided for aminoglycosides. Misfolded proteins induced by gentamicin first insert into the membrane in E. coli and lead the activation of the response regulator ArcA through CpxA, the envelope stress response sensor. ArcA then activates the tricarboxylic acid (TCA) cycle enzymes, leading to dysfunction of TCA cycle and the hyperactivation of respiration (Kohanski et al., 2008). Similar dysfunction of TCA cycle can also be observed in quinolones and $\beta$-lactams treatments, suggesting that such metabolic flux could be a shared mechanism pushing the cell into a state that provokes oxidative stress in antibiotic treatments (Kohanski et al., 2008; Belenky et al., 2015). While $\beta$-lactams may directly activate envelope stress response by affecting membrane integrity, specific triggers for quinolones remain to be worked out. Several studies found that programmed cell death mediated by YihE kinase and MazF toxin was linked to a ROS cascade in quinolone treatment (Drlica et al., 2008; DorseyOresto et al., 2013). Recently, additional evidence suggested that quinolones disrupted the nucleotide pool, leading to the increase of ATP demand (Yang et al., 2019). The increasing ATP demand elevates TCA cycle activity and cellular respiration and enhances antibiotic lethality.

\section{EXOGENOUS ROS-INDUCED KILLING DEPENDS ON A VARIETY OF INNATE MECHANISMS}

Exogenous ROS, as an antimicrobial weapon wielded by phagocytes, are generated from NADPH oxidase (NOX2) in response to microbe recognition (Panday et al., 2015). However, it has been surprisingly difficult to figure out exactly how phagocytic ROS production suppresses microbial growth (Imlay, 2019). Some indirect evidence support the notion that phagocytic ROS directly kill pathogens. Chronic granulomatous disease (CGD) is a genetic disorder in which patients lack functional NOX2 protein and therefore are associated with impaired respiratory burst (Nguyen et al., 2017). Indeed, a severe neutrophil killing defect was reported in CGD patients (Dinauer, 2005; Klebanoff, 2005). The host susceptibility to various pathogens including Salmonella enterica, Staphylococcus aureus, and Burkholderia cepacia in CGD patients highlights the importance of the respiratory burst to infectious diseases. However, whether host-derived ROS could directly kill pathogens is still a matter of debate, since several studies argued that intracellular ROS level in phagosomes was insufficient to kill pathogens (Slauch, 2011; Li and Imlay, 2018; Imlay, 2019).

Alternatively, ROS generated by NOX, as a signal, promote pathogen elimination via activating a variety of innate and adaptive mechanisms in the host cell. For example, ROS are commonly believed to be required in autophagy. NOXderived ROS are indispensable for the recruitment of the autophagy protein light chain 3 (LC3), promoting antibacterial autophagy (Huang et al., 2009; de Luca et al., 2014). In addition, during infection, neutrophils and phagocytes can activate an additional antimicrobial mechanism, referred to as neutrophil extracellular traps (NETs) (Stoiber et al., 2015). NETs, formed by chromatin and associated proteins, trap and kill various extracellular pathogens. NET formation requires the production of ROS (Papayannopoulos et al., 2010) and is impaired in NOX2-deficient neutrophils (Stoiber et al., 2015). Another study suggested that fosfomycin, a broad-spectrum antibacterial agent, could enhance NET-mediated killing of S. aureus via NOX2-dependent ROS accumulation in mouse model (Shen et al., 2016).

In addition to the NOX complex, the mitochondrion is another cellular source of ROS in immune cells (Abuaita et al., 2018). Upon macrophage activation, mitochondrial conditions favor reverse electron transport in the electron transport chain and thus generate ROS (Garaude et al., 2016; Pinegin et al., 2018). Mice deficient in proteins responsible for generation of mitoROS are highly susceptible to infections caused by Salmonella typhimurium and Listeria monocytogenes (Sonoda et al., 2007; West et al., 2011). During methicillin-resistant S. aureus (MRSA) infection, mitoROS were generated and 
delivered directly to the phagosome by mitochondrial-derived vesicles (MDVs) in a Toll-like receptor signaling-dependent manner (Abuaita et al., 2018). Of note, like NOX-mediated ROS, which can modulate IL- $1 \beta$ expression (Warnatsch et al., 2017), mitoROS can also modulate the antimicrobial functions of innate immune cells by regulating production of multiple cytokines both in vitro and in vivo (Mills et al., 2016; Herb et al., 2019).

\section{BACTERIAL RESPONSE TO OXIDATIVE STRESS: ANTIOXIDANT AND METABOLIC DEFENSES}

The systems in bacteria that regulate the expression of antioxidant defense networks have been extensively reviewed (Ezraty et al., 2017; Reniere, 2018). The ROS response is under the control of the master regulators. Transcription factors such as OxyR (i.e., in S. enterica, Francisella tularensis, and Porphyromonas gingivalis), PerR (i.e., in S. aureus and Bacillus subtilis), OhrR (i.e., in B. subtilis and Mycobacterium smegmatis), and SoxRS (i.e., in E. coli and S. aureus) can be activated by direct oxidation of their sensor proteins and then adjusting the bacterial response appropriately (Chiang and Schellhorn, 2012). Although the particulars vary among different species, in general, these regulons regulate genes required for antioxidant defense, including superoxide dismutase, catalase, thioredoxins, heme biosynthesis machinery, glutathione reductases, ferric uptake regulator (Fur), ferritin, and bacterioferritin (Imlay, 2008; Chiang and Schellhorn, 2012; Reniere, 2018). In addition, iron homeostasis is also critical to mitigate redox damage induced by Fenton reaction. Therefore, in pathogenic bacteria (i.e., E. coli, S. aureus, and Salmonella), the iron-sensing transcriptional repressors, such as Fur and DtxR (diphtheria toxin repressor), can also be utilized to sustain redox homeostasis by controlling the expression of genes encoding iron acquisition systems and iron-dependent enzymes (Troxell and Hassan, 2013).

Apart from antioxidant defense systems, metabolism remodeling also plays a pivotal role in mitigating oxidative damage (Figure 1). Metabolic adaptions can reduce oxidative burden by retarding respiration. For example, the glyoxylate shunt (GS) is an anaplerotic reaction of the TCA cycle developed in numerous species, which bypasses two NADHgenerating steps (Lemire et al., 2017; Dolan and Welch, 2018). In B. cepacia, GS genes were upregulated in cells surviving aminoglycoside treatment (Van Acker et al., 2013). Similarly, in Mycobacterium tuberculosis (MTB), GS enzyme isocitrate lyase deficient mutants were significantly more susceptible than wild-type strain toward isoniazid, rifampicin, and streptomycin (Nandakumar et al., 2014). Consistent with these observations, the Collins group found that glyoxylate could serve as a direct biochemical inducer of aminoglycoside tolerance via reducing cellular respiration. Meanwhile, TCA cycle intermediates, such as fumarate, significantly potentiate tobramycin lethality (Meylan et al., 2017).
Replenishment of antioxidants can also be achieved by metabolic modulation. Pentose phosphate (PP) pathway is an important target to mitigate ROS damage, since $\mathrm{NADPH}$ is the cofactor for antioxidant systems. In E. coli, by increased abundance of glucose-6-phosphate dehydrogenase, the metabolic flux can be rerouted toward the PP pathway, leading to increased ROS tolerance (Christodoulou et al., 2018).

Ketoacids including $\alpha$-ketoglutarate $(\mathrm{KG})$ and pyruvate can undergo non-enzymatic decarboxylation in the presence of ROS, generate non-toxic byproducts, and thus alleviate the ROS damage. Bacteria like E. coli and Pseudomonas fluorescens tend to pool ketoacids both inside the cell and in the extracellular matrix under ROS conditions. For example, the increased generation of KG can be achieved by the modulation of TCA cycle enzymes like isocitrate dehydrogenase when using citrate, glucose, and malate as carbon sources (Alhasawi et al., 2016). It can also be achieved by the deamination of glutamate when glutamate or histidine is supplied as the carbon source (Lemire et al., 2010, 2017). When exposed to $\mathrm{H}_{2} \mathrm{O}_{2}, P$. fluorescens can go through a metabolic reconfiguration with enhanced activity of substrate-level phosphorylation as well as impaired activity of the TCA cycle, leading to evident pyruvate synthesis with glucose or glycerol as the carbon source (Bignucolo et al., 2013; Alhasawi et al., 2016).

\section{INDUCTION OF BACTERIAL VIRULENCE UNDER ROS CONDITIONS}

In addition to antioxidant defenses, activations of the oxidative defense regulators are also required for full virulence in pathogens. For example, OxyR contributes to the virulence of E. coli and Pseudomonas aeruginosa (Lau et al., 2005; Wei et al., 2012; Fang et al., 2016). Similarly, SoxRS has been shown to act as a positive regulator in Salmonella pathogenicity island (SPI)2 mediated virulence in S. enterica (Wang P. et al., 2020). In $S$. aureus, after oxidation of the sensor protein by ROS, the redoxsignaling regulator AirSR positively regulates the biosynthesis of staphyloxanthin (STX), an important virulence factor of S. aureus (Hall et al., 2017). MntR, a member of the DtxR family, is required for $S$. aureus pathogenesis via maintaining manganese homeostasis (Grunenwald et al., 2019).

In host conditions, virulence modulation can be achieved by ROS indirectly. During ROS generation, phagocytes simultaneously produce glutathione (GSH) to sustain intracellular redox homeostasis. Certain intracellular pathogens have even evolved to exploit the host GSH for adaptions. In Burkholderia pseudomallei, virulence is completely dependent on the expression of a type VI secretion system (T6SS). During the exit from phagosome, $B$. pseudomallei senses host GSH via sensor protein VirA, which then activates the expression of the T6SS (Wong et al., 2015). In L. monocytogenes, the extracellular host GSH can trigger the production of bacterial GSH. Both host GSH and bacterial GSH bind to the master virulence regulator PrfA and function as an allosteric activator (Reniere et al., 2015; Portman et al., 2017). 


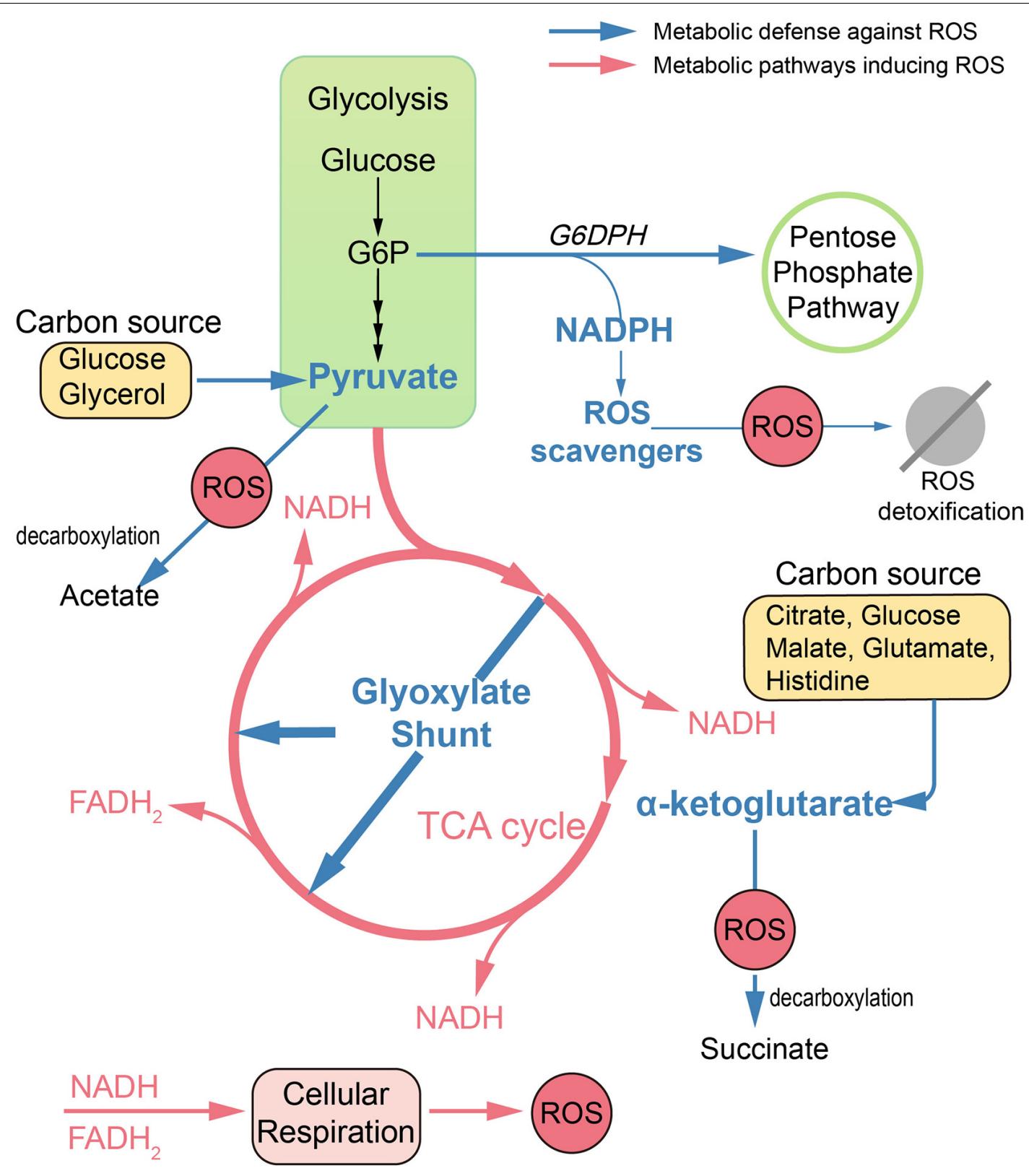

FIGURE 1 | Metabolic remodeling designed to reduce oxidative damage. In antibiotic treatments, dysfunction of TCA cycle and hyperactivation of cell respiration lead to ROS accumulation in bacteria. Defense against ROS can be achieved by metabolic remodeling. Reduced endogenous ROS formation can be achieved by upregulation of the glyoxylate shunt, which bypasses two NADH-generating steps. Replenishment of antioxidants can be achieved by rerouting metabolism toward the pentose phosphate pathway and enhancing production of cofactor NADPH. Also, ketoacids, including pyruvate and $\alpha$-ketoglutarate, can mitigate oxidative damage via non-enzymatic decarboxylation in the presence of numerous carbon sources. G6P, glucose 6-phosphate; G6DPH, glucose-6-phosphate dehydrogenase.

\section{THRIVE UNDER ROS CONDITIONS BY METABOLIC REMODELING}

While pathogens can be eliminated by ROS, it is quite clear that certain pathogens exploit ROS to coordinate metabolism to thrive. For example, S. typhimurium evidently makes use of hostderived ROS during intestinal inflammation. ROS generated by phagocytes convert thiosulfate to tetrathionate, which in turn can be used as a respiratory electron sink by S. typhimurium, allowing it to outcompete the native microbiota (Winter et al., 2010; Bäumler and Sperandio, 2016). Another example comes from E. coli. In the intestinal lumen, oxygen from the epithelium collides with sulfide generated by luminal bacteria, potentially generating $\mathrm{H}_{2} \mathrm{O}_{2}$ through direct reaction. Also, lactic-acid bacteria excrete $\mathrm{H}_{2} \mathrm{O}_{2}$ as a direct metabolic product. Under such circumstances, cytochrome $c$ peroxidase (Ccp) regulated 


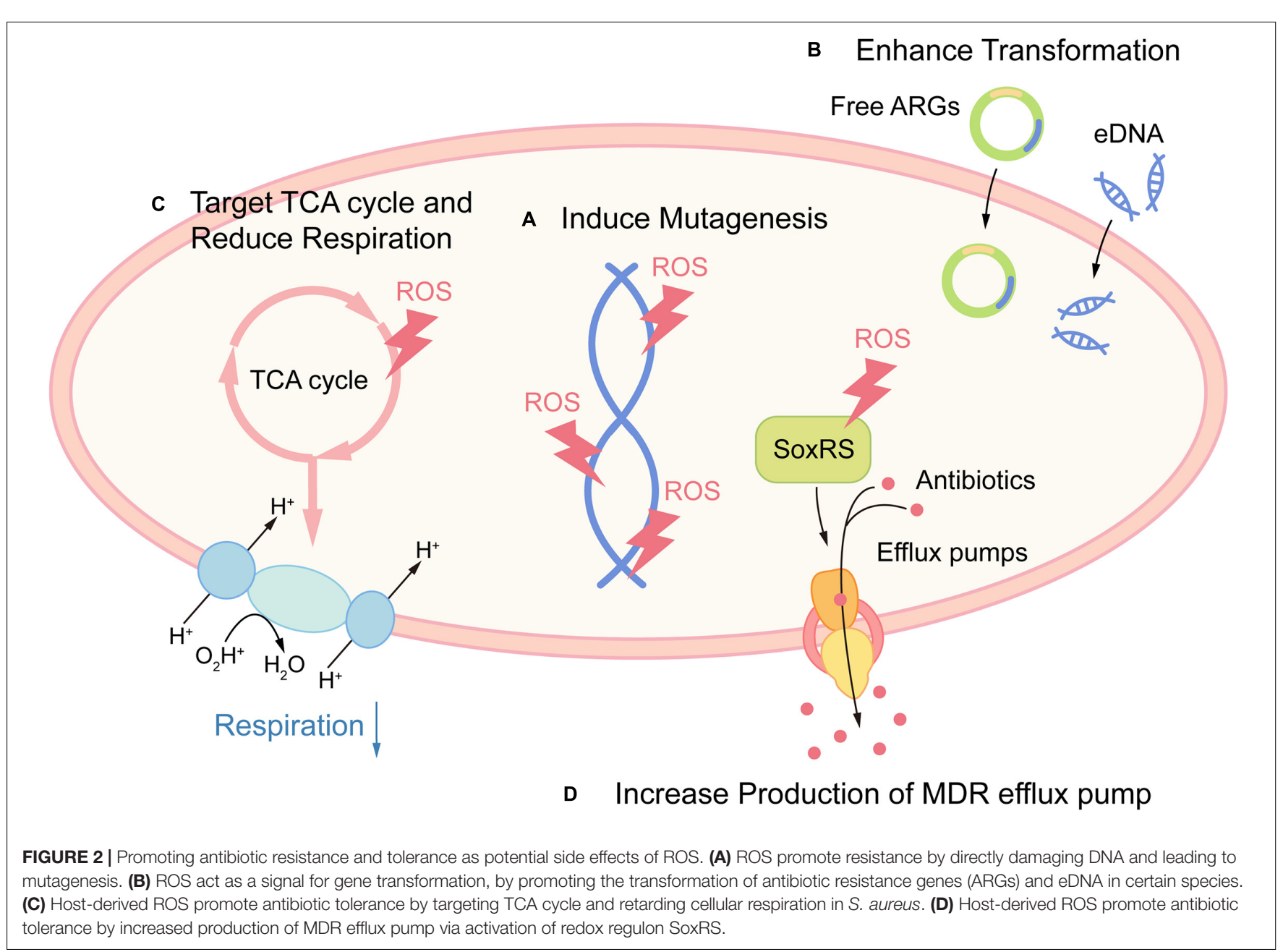

by OxyR allows E. coli to employ $\mathrm{H}_{2} \mathrm{O}_{2}$ as a terminal oxidant for respiration (Khademian and Imlay, 2017). Helicobacter pylori is well known as a ROS-inducing gastric pathogen. It utilizes chemotaxis to seek sites optimal for efficient colonization. Recent studies showed that ROS could be sensed in $H$. pylori by the chemoreceptor TlpD. Host oxidants hypochlorous acid ( $\mathrm{HOCl})$ could act as a chemoattractant by reversibly oxidizing TlpD that inactivates the chemotransduction signaling complex (Perkins et al., 2019). While $\mathrm{H}_{2} \mathrm{O}_{2}$ could act as a chemorepellent which initiates chemotaxis through TlpD to promote gastric gland colonization (Collins et al., 2018).

\section{BACTERIAL RESISTANCE AND TOLERANCE AS SIDE EFFECTS OF ROS}

Recently, the radical-based theory in pathogen clearance has been questioned for the protective role of ROS against antimicrobial killing (Burger and Drlica, 2009; Mosel et al., 2013). ROS can directly damage DNA, leading to genetic mutations. Indeed, ROS produced by non-lethal antibiotics induce mutations in specific antibiotic targets and promote the development of multi-drug resistance (Kohanski et al., 2010a; Takahashi et al., 2017). If ROS is a shared mechanism in antibiotic lethality, then it is to be expected that protection against ROS can be one of the shared traits for bacterial resistance against antibiotics as well. Indeed, bactericidal antibiotic mediates de novo acquisition of resistance, which then provides protection against ROS accumulation upon exposure to a different type of antibiotics (Hoeksema et al., 2018). In addition, it was recently demonstrated that non-lethal exposure to $\mathrm{H}_{2} \mathrm{O}_{2}$ boosted evolvability of bacterial populations by enhancing survival under oxidative stress (Rodriguez-Rojas et al., 2020).

ROS also act as a signal for gene transformation. $\mathrm{H}_{2} \mathrm{O}_{2}$ is a metabolic product of certain oral streptococci such as Streptococcus gordonii under aerobic conditions. In $\mathrm{H}_{2} \mathrm{O}_{2}$ producing streptococci, the release of extracellular DNA (eDNA) is entirely dependent on $\mathrm{H}_{2} \mathrm{O}_{2}$. Also, numerous $\mathrm{H}_{2} \mathrm{O}_{2}$-nonproducing species like Streptococcus mutans and Streptococcus pyogenes release eDNA in a $\mathrm{H}_{2} \mathrm{O}_{2}$-dependent manner (Itzek et al., 2011), suggesting that ROS serve as an important environmental signal in oral biofilm (Redanz et al., 2018). Intriguingly, a recent study shows that non-antibiotic pharmaceuticals including non-steroidal anti-inflammatory drugs (NSAIDs) and the lipid-lowering drug also induce significant ROS accumulation in Acinetobacter baylyi, which is closely related to the enhanced transformation of antibiotic resistance genes (ARGs) (Wang Y. et al., 2020). 
ROS are key weapons in host cells, while recent evidence suggests that host ROS induce antibiotic tolerance during infection. ROS generated by macrophages target TCA enzymes aconitase and succinate dehydrogenase and coerce $S$. aureus into a metabolic state with reduced respiration, which is incompatible with the killing mechanism of most bactericidal antibiotics (Rowe et al., 2019). The persister is an extreme case of tolerance for the high levels of multi-drug tolerance (Brauner et al., 2016). Host ROS can also modulate persister formation in E. coli. The activation of redox regulators SoxRS results in increasing expression of the AcrAB-TolC multidrug-resistant (MDR) pump, which in turn lowers the fluoroquinolones concentration and promotes antibiotic tolerance (Wu et al., 2012).

Together, these examples indicate that ROS may potentiate the emergence of bacterial resistance and tolerance (Figure 2). Although there is currently no clinical evidence proving this hypothesis, contradictory evidence favoring these potential side effects have been indicated in several studies. Early study suggested that $S$. aureus internalized in leucocytes isolated from patients with CGD (with impaired respiratory burst) were more susceptible to rifampicin, when compared with wild-type leucocytes (Jacobs and Wilson, 1983). Also, elevated levels of oxidation products are related to MTB infection in pulmonary tuberculosis (PTB) patients (Amaral et al., 2016). While there is scarce demonstration that the use of antioxidants can increase pathogen burden, a study showed that the antioxidant resveratrol reduced Serratia marcescens burden in mice (Lu et al., 2008). Surprisingly, the use of antioxidant $\mathrm{N}$-acetyl cysteine (NAC) as an adjuvant to directly observed treatment short course significantly caused early sputum negativity in PTB patients (Mahakalkar et al., 2017).

\section{NOVEL ANTIMICROBIAL STRATEGIES CENTERED AROUND ROS}

The efficacy of antibiotics has been endangered by the rapid emergence of resistant pathogens. Therefore, new approaches to fight against pathogens, including antimicrobial photodynamic therapy (aPDT) and cold atmospheric plasma (CAP), have been suggested as efficient alternative approaches. Although their antibacterial efficacy also centered around ROS, the modes of action for these new techniques are distinct from conventional antibiotics, as ROS-mediated damage in such therapies is the primary stress-induced damage (Wilson and Patterson, 2008; Vatansever et al., 2013). For example, aPDT uses photosensitizers

\section{REFERENCES}

Abuaita, B. H., Schultz, T. L., and O’Riordan, M. X. (2018). Mitochondria-derived vesicles deliver antimicrobial reactive oxygen species to control phagosomelocalized Staphylococcus aureus. Cell Host Microbe 24, 625-636.e5. doi: 10.1016/ j.chom.2018.10.005

Alhasawi, A., Thomas, S. C., and Appanna, V. D. (2016). Metabolic networks to generate pyruvate, PEP and ATP from glycerol in Pseudomonas fluorescens. Enzyme Microb. Technol. 85, 51-56. doi: 10.1016/j.enzmictec.2016.01.007

Amaral, E. P., Conceição, E. L., Costa, D. L., Rocha, M. S., Marinho, J. M., CordeiroSantos, M., et al. (2016). N-acetyl-cysteine exhibits potent anti-mycobacterial
(PS) to generate ROS upon irradiation by visible light at specific wavelength, and CAPs are partly ionized gases producing a reactive mix by interacting with oxygen, generating a cocktail of ROS (Brany et al., 2020). Due to direct redox-active properties, aPDT and CAP can cause multi-target oxidative damage to pathogens (Melo et al., 2013; Hu et al., 2018) as well as direct oxidation of polysaccharides in biofilm (Beirão et al., 2014; Jiao et al., 2019). Due to their multi-target mode of action and large quantities of ROS production which overwhelm the antioxidant defenses in pathogens, the additional advantage of these new techniques is the lack of development of resistance mechanisms (Tavares et al., 2010; Kvam et al., 2012). As novel ROSinducing strategies, aPDT and CAP showed excellent potential to tackle difficult-to-eradicate infections as alternative treatments in wound healing, dental cure, and food decontamination (Wilson and Patterson, 2008; Rao et al., 2020).

\section{CONCLUDING REMARKS}

ROS are attractive weapons to kill pathogenic microbial cells. However, ROS, as a double-edged sword, should be regulated with care since under non-lethal ROS, certain pathogens have evolved delicate mechanisms to utilize ROS for their adaption to thrive. Also, numerous pathogens can use ROS as a stepstone for the evolution of antibiotic tolerance and resistance. Efforts to target bacterial adaptive pathways, as well as the use of novel ROS-inducing antibacterial strategies, will be promising approaches for antibacterial therapy.

\section{AUTHOR CONTRIBUTIONS}

HL, XZ, YH, BL, LC, and BR: conception/design of the work, agreement to be accountable for all aspects of the work. HL, LC, and BR: drafting the work. XZ, LC, and BR: final approval of the manuscript to be published. All authors contributed to the article and approved the submitted version.

\section{FUNDING}

This study was supported by the National Natural Science Foundation of China grants 81870778 (BR), 81600858 (BR), and 82071106 (LC) and the Applied Basic Research Programs of Sichuan Province 2020YJ0227 (BR).

activity in addition to its known anti-oxidative functions. BMC Microbiol. 16:251. doi: 10.1186/s12866-016-0872-7

Bäumler, A. J., and Sperandio, V. (2016). Interactions between the microbiota and pathogenic bacteria in the gut. Nature 535, 85-93. doi: 10.1038/nature18849

Beirão, S., Fernandes, S., Coelho, J., Faustino, M. A., Tomé, J. P., Neves, M. G., et al. (2014). Photodynamic inactivation of bacterial and yeast biofilms with a cationic porphyrin. Photochem. Photobiol. 90, 1387-1396. doi: 10.1111/php. 12331

Belenky, P., Ye, J. D., Porter, C. B., Cohen, N. R., Lobritz, M. A., Ferrante, T., et al. (2015). Bactericidal antibiotics induce toxic metabolic perturbations that lead to cellular damage. Cell Rep. 13, 968-980. doi: 10.1016/j.celrep.2015.09.059 
Bignucolo, A., Appanna, V. P., Thomas, S. C., Auger, C., Han, S., Omri, A., et al. (2013). Hydrogen peroxide stress provokes a metabolic reprogramming in Pseudomonas fluorescens: enhanced production of pyruvate. J. Biotechnol. 167, 309-315. doi: 10.1016/j.jbiotec.2013.07.002

Brany, D., Dvorska, D., Halasova, E., and Skovierova, H. (2020). Cold atmospheric plasma: a powerful tool for modern medicine. Int. J. Mol. Sci. 21:2932. doi: 10.3390/ijms21082932

Brauner, A., Fridman, O., Gefen, O., and Balaban, N. Q. (2016). Distinguishing between resistance, tolerance and persistence to antibiotic treatment. Nat. Rev. Microbiol. 14, 320-330. doi: 10.1038/nrmicro.2016.34

Burger, R. M., and Drlica, K. (2009). Superoxide protects Escherichia coli from bleomycin mediated lethality. J. Inorg. Biochem. 103, 1273-1277. doi: 10.1016/j. jinorgbio.2009.07.009

Chiang, S. M., and Schellhorn, H. E. (2012). Regulators of oxidative stress response genes in Escherichia coli and their functional conservation in bacteria. Arch. Biochem. Biophys. 525, 161-169. doi: 10.1016/j.abb.2012.02.007

Christodoulou, D., Link, H., Fuhrer, T., Kochanowski, K., Gerosa, L., and Sauer, U. (2018). Reserve flux capacity in the pentose phosphate pathway enables Escherichia coli's rapid response to oxidative stress. Cell Syst. 6, 569-578.e7. doi: 10.1016/j.cels.2018.04.009

Collins, K. D., Hu, S., Grasberger, H., Kao, J. Y., and Ottemann, K. M. (2018). Chemotaxis allows bacteria to overcome host-generated reactive oxygen species that constrain gland colonization. Infect. Immun. 86:e00878-17.

de Luca, A., Smeekens, S. P., Casagrande, A., Iannitti, R., Conway, K. L., Gresnigt, M. S., et al. (2014). IL-1 receptor blockade restores autophagy and reduces inflammation in chronic granulomatous disease in mice and in humans. Proc. Natl. Acad. Sci. U.S.A. 111, 3526-3531. doi: 10.1073/pnas.13228 31111

Dinauer, M. C. (2005). Chronic granulomatous disease and other disorders of phagocyte function. Hematology Am. Soc. Hematol. Educ. Program 2005, 89-95. doi: 10.1182/asheducation-2005.1.89

Dolan, S. K., and Welch, M. (2018). The glyoxylate shunt, 60 years on. Annu. Rev. Microbiol. 72, 309-330. doi: 10.1146/annurev-micro-090817-062257

Dorsey-Oresto, A., Lu, T., Mosel, M., Wang, X., Salz, T., Drlica, K., et al. (2013). YihE kinase is a central regulator of programmed cell death in bacteria. Cell Rep. 3, 528-537. doi: 10.1016/j.celrep.2013.01.026

Drlica, K., Malik, M., Kerns, R. J., and Zhao, X. (2008). Quinolone-mediated bacterial death. Antimicrob. Agents Chemother. 52, 385-392. doi: 10.1128/aac. 01617-06

Dwyer, D. J., Kohanski, M. A., Hayete, B., and Collins, J. J. (2007). Gyrase inhibitors induce an oxidative damage cellular death pathway in Escherichia coli. Mol. Syst. Biol. 3:91. doi: 10.1038/msb4100135

Ezraty, B., Gennaris, A., Barras, F., and Collet, J. F. (2017). Oxidative stress, protein damage and repair in bacteria. Nat. Rev. Microbiol. 15, 385-396. doi: 10.1038/ nrmicro.2017.26

Fang, F. C., Frawley, E. R., Tapscott, T., and Vazquez-Torres, A. (2016). Bacterial stress responses during host infection. Cell Host Microbe 20, 133-143. doi: 10.1016/j.chom.2016.07.009

Garaude, J., Acín-Pérez, R., Martínez-Cano, S., Enamorado, M., Ugolini, M., Nistal-Villán, E., et al. (2016). Mitochondrial respiratory-chain adaptations in macrophages contribute to antibacterial host defense. Nat. Immunol. 17, 1037-1045. doi: 10.1038/ni.3509

Grunenwald, C. M., Choby, J. E., Juttukonda, L. J., Beavers, W. N., Weiss, A., Torres, V. J., et al. (2019). Manganese detoxification by MntE is critical for resistance to oxidative stress and virulence of Staphylococcus aureus. mBio 10:e02915-18.

Hall, J. W., Yang, J., Guo, H., and Ji, Y. (2017). The Staphylococcus aureus AirSR two-component system mediates reactive oxygen species resistance via transcriptional regulation of staphyloxanthin production. Infect. Immun. 85:e00838-16.

Herb, M., Gluschko, A., Wiegmann, K., Farid, A., Wolf, A., Utermöhlen, O., et al. (2019). Mitochondrial reactive oxygen species enable proinflammatory signaling through disulfide linkage of NEMO. Sci. Signal. 12:eaar5926. doi: 10.1126/scisignal.aar5926

Hoeksema, M., Brul, S., and Ter Kuile, B. H. (2018). Influence of reactive oxygen species on de novo acquisition of resistance to bactericidal antibiotics. Antimicrob. Agents Chemother. 62:e2354-17.
Hong, Y., Zeng, J., Wang, X., Drlica, K., and Zhao, X. (2019). Post-stress bacterial cell death mediated by reactive oxygen species. Proc. Natl. Acad. Sci. U.S.A. 116, 10064-10071. doi: 10.1073/pnas.1901730116

Hu, X., Huang, Y.-Y., Wang, Y., Wang, X., and Hamblin, M. R. (2018). Antimicrobial photodynamic therapy to control clinically relevant biofilm infections. Front. Microbiol. 9:1299. doi: 10.3389/fmicb.2018.01299

Huang, J., Canadien, V., Lam, G. Y., Steinberg, B. E., Dinauer, M. C., Magalhaes, M. A., et al. (2009). Activation of antibacterial autophagy by NADPH oxidases. Proc. Natl. Acad. Sci. U.S.A. 106, 6226-6231. doi: 10.1073/pnas.0811045106

Imlay, J. A. (2008). Cellular defenses against superoxide and hydrogen peroxide. Annu. Rev. Biochem. 77, 755-776. doi: 10.1146/annurev.biochem.77.061606. 161055

Imlay, J. A. (2015). Diagnosing oxidative stress in bacteria: not as easy as you might think. Curr. Opin. Microbiol. 24, 124-131. doi: 10.1016/j.mib.2015.01.004

Imlay, J. A. (2019). Where in the world do bacteria experience oxidative stress? Environ. Microbiol. 21, 521-530. doi: 10.1111/1462-2920.14445

Itzek, A., Zheng, L., Chen, Z., Merritt, J., and Kreth, J. (2011). Hydrogen peroxide-dependent DNA release and transfer of antibiotic resistance genes in Streptococcus gordonii. J. Bacteriol. 193, 6912-6922. doi: 10.1128/jb.05791-11

Jacobs, R. F., and Wilson, C. B. (1983). Activity of antibiotics in chronic granulomatous disease leukocytes. Pediatr. Res. 17, 916-919. doi: 10.1203/ 00006450-198311000-00016

Jiao, Y., Tay, F. R., Niu, L. N., and Chen, J. H. (2019). Advancing antimicrobial strategies for managing oral biofilm infections. Int. J. Oral Sci. 11:28.

Keren, I., Wu, Y., Inocencio, J., Mulcahy, L. R., and Lewis, K. (2013). Killing by bactericidal antibiotics does not depend on reactive oxygen species. Science 339, 1213-1216. doi: 10.1126/science. 1232688

Khademian, M., and Imlay, J. A. (2017). Escherichia coli cytochrome c peroxidase is a respiratory oxidase that enables the use of hydrogen peroxide as a terminal electron acceptor. Proc. Natl. Acad. Sci. U.S.A. 114, E6922-E6931. doi: 10.1073/ pnas. 1701587114

Klebanoff, S. J. (2005). Myeloperoxidase: friend and foe. J. Leukoc. Biol. 77, 598-625. doi: 10.1189/jlb.1204697

Kohanski, M. A., DePristo, M. A., and Collins, J. J. (2010a). Sublethal antibiotic treatment leads to multidrug resistance via radical-induced mutagenesis. Mol. Cell 37, 311-320. doi: 10.1016/j.molcel.2010.01.003

Kohanski, M. A., Dwyer, D. J., and Collins, J. J. (2010b). How antibiotics kill bacteria: from targets to networks. Nat. Rev. Microbiol. 8, 423-435. doi: 10.1038/ nrmicro2333

Kohanski, M. A., Dwyer, D. J., Hayete, B., Lawrence, C. A., and Collins, J. J. (2007). A common mechanism of cellular death induced by bactericidal antibiotics. Cell 130, 797-810. doi: 10.1016/j.cell.2007.06.049

Kohanski, M. A., Dwyer, D. J., Wierzbowski, J., Cottarel, G., and Collins, J. J. (2008). Mistranslation of membrane proteins and two-component system activation trigger antibiotic-mediated cell death. Cell 135, 679-690. doi: 10.1016/j.cell. 2008.09.038

Kvam, E., Davis, B., Mondello, F., and Garner, A. L. (2012). Nonthermal atmospheric plasma rapidly disinfects multidrug-resistant microbes by inducing cell surface damage. Antimicrob. Agents Chemother. 56, 2028-2036. doi: 10.1128/aac.05642-11

Lau, G. W., Britigan, B. E., and Hassett, D. J. (2005). Pseudomonas aeruginosa OxyR is required for full virulence in rodent and insect models of infection and for resistance to human neutrophils. Infect. Immun. 73, 2550-2553. doi: 10.1128/iai.73.4.2550-2553.2005

Lemire, J., Alhasawi, A., Appanna, V. P., Tharmalingam, S., and Appanna, V. D. (2017). Metabolic defence against oxidative stress: the road less travelled so far. J. Appl. Microbiol. 123, 798-809. doi: 10.1111/jam.13509

Lemire, J., Milandu, Y., Auger, C., Bignucolo, A., Appanna, V. P., and Appanna, V. D. (2010). Histidine is a source of the antioxidant, alpha-ketoglutarate, in Pseudomonas fluorescens challenged by oxidative stress. FEMS Microbiol. Lett. 309, 170-177. doi: 10.1111/j.1574-6968.2010.02034.x

Li, X., and Imlay, J. A. (2018). Improved measurements of scant hydrogen peroxide enable experiments that define its threshold of toxicity for Escherichia coli. Free Radic. Biol. Med. 120, 217-227. doi: 10.1016/j.freeradbiomed.2018.03.025

Lobritz, M. A., Belenky, P., Porter, C. B., Gutierrez, A., Yang, J. H., Schwarz, E. G., et al. (2015). Antibiotic efficacy is linked to bacterial cellular respiration. Proc. Natl. Acad. Sci. U.S.A. 112, 8173-8180. doi: 10.1073/pnas.1509743112 
Lu, C. C., Lai, H. C., Hsieh, S. C., and Chen, J. K. (2008). Resveratrol ameliorates Serratia marcescens-induced acute pneumonia in rats. J. Leukoc. Biol. 83, 1028-1037. doi: 10.1189/jlb.0907647

Luan, G., Hong, Y., Drlica, K., and Zhao, X. (2018). Suppression of reactive oxygen species accumulation accounts for paradoxical bacterial survival at high quinolone concentration. Antimicrob. Agents Chemother. 62:e01622-17.

Mahakalkar, S. M., Nagrale, D., Gaur, S., Urade, C., Murhar, B., and Turankar, A. (2017). N-acetylcysteine as an add-on to Directly Observed Therapy Short-I therapy in fresh pulmonary tuberculosis patients: a randomized, placebocontrolled, double-blinded study. Perspect. Clin. Res. 8, 132-136. doi: 10.4103/ 2229-3485.210450

Melo, T., Santos, N., Lopes, D., Alves, E., Maciel, E., Faustino, M. A., et al. (2013). Photosensitized oxidation of phosphatidylethanolamines monitored by electrospray tandem mass spectrometry. J. Mass Spectrom. 48, 1357-1365. doi: 10.1002/jms.3301

Meylan, S., Porter, C. B. M., Yang, J. H., Belenky, P., Gutierrez, A., Lobritz, M. A., et al. (2017). Carbon sources tune antibiotic susceptibility in Pseudomonas aeruginosa via tricarboxylic acid cycle control. Cell Chem. Biol. 24, 195-206. doi: 10.1016/j.chembiol.2016.12.015

Mills, E. L., Kelly, B., Logan, A., Costa, A. S. H., Varma, M., Bryant, C. E., et al. (2016). Succinate dehydrogenase supports metabolic repurposing of mitochondria to drive inflammatory macrophages. Cell 167, 457-470.e13. doi: 10.1016/j.cell.2016.08.064

Mittler, R. (2017). ROS are good. Trends Plant Sci. 22, 11-19. doi: 10.1016/j.tplants. 2016.08.002

Mosel, M., Li, L., Drlica, K., and Zhao, X. (2013). Superoxide-mediated protection of Escherichia coli from antimicrobials. Antimicrob. Agents Chemother. 57, 5755-5759. doi: 10.1128/aac.00754-13

Nandakumar, M., Nathan, C., and Rhee, K. Y. (2014). Isocitrate lyase mediates broad antibiotic tolerance in Mycobacterium tuberculosis. Nat. Commun. 5:4306. doi: $10.1038 /$ ncomms5306

Nguyen, G. T., Green, E. R., and Mecsas, J. (2017). Neutrophils to the ROScue: mechanisms of NADPH oxidase activation and bacterial resistance. Front. Cell. Infect. Microbiol. 7:373. doi: 10.3389/fcimb.2017.00373

Panday, A., Sahoo, M. K., Osorio, D., and Batra, S. (2015). NADPH oxidases: an overview from structure to innate immunity-associated pathologies. Cell. Mol. Immunol. 12, 5-23. doi: 10.1038/cmi.2014.89

Papayannopoulos, V., Metzler, K. D., Hakkim, A., and Zychlinsky, A. (2010). Neutrophil elastase and myeloperoxidase regulate the formation of neutrophil extracellular traps. J. Cell Biol. 191, 677-691. doi: 10.1083/jcb.201006052

Perkins, A., Tudorica, D. A., Amieva, M. R., Remington, S. J., and Guillemin, K. (2019). Helicobacter pylori senses bleach $(\mathrm{HOCl})$ as a chemoattractant using a cytosolic chemoreceptor. PLoS Biol. 17:e3000395. doi: 10.1371/journal.pbio. 3000395

Pinegin, B., Vorobjeva, N., Pashenkov, M., and Chernyak, B. (2018). The role of mitochondrial ROS in antibacterial immunity. J. Cell. Physiol. 233, 3745-3754. doi: $10.1002 /$ jcp. 26117

Portman, J. L., Dubensky, S. B., Peterson, B. N., Whiteley, A. T., and Portnoy, D. A. (2017). Activation of the Listeria monocytogenes virulence program by a reducing environment. $m$ Bio 8:e01595-17.

Rao, Y., Shang, W., Yang, Y., Zhou, R., and Rao, X. (2020). Fighting mixed-species microbial biofilms with cold atmospheric plasma. Front. Microbiol. 11:1000. doi: $10.3389 /$ fmicb. 2020.01000

Redanz, S., Cheng, X., Giacaman, R. A., Pfeifer, C. S., Merritt, J., and Kreth, J. (2018). Live and let die: hydrogen peroxide production by the commensal flora and its role in maintaining a symbiotic microbiome. Mol. Oral Microbiol. 33, 337-352. doi: 10.1111/omi.12231

Reniere, M. L. (2018). Reduce, induce, thrive: bacterial redox sensing during pathogenesis. J. Bacteriol. 200:e00128-18.

Reniere, M. L., Whiteley, A. T., Hamilton, K. L., John, S. M., Lauer, P., Brennan, R. G., et al. (2015). Glutathione activates virulence gene expression of an intracellular pathogen. Nature 517, 170-173. doi: 10.1038/nature14029

Rodriguez-Rojas, A., Kim, J. J., Johnston, P. R., Makarova, O., Eravci, M., Weise, C., et al. (2020). Non-lethal exposure to H2O2 boosts bacterial survival and evolvability against oxidative stress. PLoS Genet. 16:e1008649. doi: 10.1371/ journal.pgen.1008649

Rowe, S. E., Wagner, N. J., Li, L., Beam, J. E., Wilkinson, A. D., Radlinski, L. C., et al. (2019). Reactive oxygen species induce antibiotic tolerance during systemic Staphylococcus aureus infection. Nat. Microbiol. 5, 282-290. doi: 10. 1038/s41564-019-0627-y

Shen, F., Tang, X., Cheng, W., Wang, Y., Wang, C., Shi, X., et al. (2016). Fosfomycin enhances phagocyte-mediated killing of Staphylococcus aureus by extracellular traps and reactive oxygen species. Sci. Rep. 6:19262. doi: 10.1038/srep 19262

Slauch, J. M. (2011). How does the oxidative burst of macrophages kill bacteria? Still an open question. Mol. Microbiol. 80, 580-583. doi: 10.1111/j.1365-2958. 2011.07612.x

Sonoda, J., Laganière, J., Mehl, I. R., Barish, G. D., Chong, L. W., Li, X., et al. (2007). Nuclear receptor ERR alpha and coactivator PGC-1 beta are effectors of IFN-gamma-induced host defense. Genes Dev. 21, 1909-1920. doi: 10.1101/ gad. 1553007

Stoiber, W., Obermayer, A., Steinbacher, P., and Krautgartner, W. D. (2015). The role of reactive oxygen species (ROS) in the formation of extracellular traps (ETs) in humans. Biomolecules 5, 702-723. doi: 10.3390/biom5020702

Stokes, J. M., Lopatkin, A. J., Lobritz, M. A., and Collins, J. J. (2019). Bacterial metabolism and antibiotic efficacy. Cell Metab. 30, 251-259. doi: 10.1016/j.cmet. 2019.06.009

Takahashi, N., Gruber, C. C., Yang, J. H., Liu, X., Braff, D., Yashaswini, C. N., et al. (2017). Lethality of MalE-LacZ hybrid protein shares mechanistic attributes with oxidative component of antibiotic lethality. Proc. Natl. Acad. Sci. U.S.A. 114, 9164-9169. doi: 10.1073/pnas.1707466114

Tavares, A., Carvalho, C. M., Faustino, M. A., Neves, M. G., Tomé, J. P., Tomé, A. C., et al. (2010). Antimicrobial photodynamic therapy: study of bacterial recovery viability and potential development of resistance after treatment. Mar. Drugs 8, 91-105. doi: 10.3390/md8010091

Troxell, B., and Hassan, H. M. (2013). Transcriptional regulation by ferric uptake regulator (Fur) in pathogenic bacteria. Front. Cell. Infect. Microbiol. 3:59. doi: $10.3389 /$ fcimb.2013.00059

Van Acker, H., and Coenye, T. (2017). The role of reactive oxygen species in antibiotic-mediated killing of bacteria. Trends Microbiol. 25, 456-466. doi: 10. 1016/j.tim.2016.12.008

Van Acker, H., Sass, A., Bazzini, S., De Roy, K., Udine, C., Messiaen, T., et al. (2013). Biofilm-grown Burkholderia cepacia complex cells survive antibiotic treatment by avoiding production of reactive oxygen species. PLoS One 8:e58943. doi: 10.1371/journal.pone.0058943

Vatansever, F., de Melo, W. C., Avci, P., Vecchio, D., Sadasivam, M., Gupta, A., et al. (2013). Antimicrobial strategies centered around reactive oxygen speciesbactericidal antibiotics, photodynamic therapy, and beyond. FEMS Microbiol. Rev. 37, 955-989. doi: 10.1111/1574-6976.12026

Wang, P., Zhang, H., Liu, Y., Lv, R., Liu, X., Song, X., et al. (2020). SoxS is a positive regulator of key pathogenesis genes and promotes intracellular replication and virulence of Salmonella Typhimurium. Microb. Pathog. 139:103925. doi: 10. 1016/j.micpath.2019.103925

Wang, Y., Lu, J., Engelstadter, J., Zhang, S., Ding, P., Mao, L., et al. (2020). Nonantibiotic pharmaceuticals enhance the transmission of exogenous antibiotic resistance genes through bacterial transformation. ISME J. 14, 2179-2196. doi: 10.1038/s41396-020-0679-2

Warnatsch, A., Tsourouktsoglou, T. D., Branzk, N., Wang, Q., Reincke, S., Herbst, S., et al. (2017). Reactive oxygen species localization programs inflammation to clear microbes of different size. Immunity 46, 421-432. doi: 10.1016/j.immuni. 2017.02.013

Wei, Q., Le Minh, P. N., Dötsch, A., Hildebrand, F., Panmanee, W., Elfarash, A., et al. (2012). Global regulation of gene expression by OxyR in an important human opportunistic pathogen. Nucleic Acids Res. 40, 4320-4333. doi: 10.1093/ nar/gks017

West, A. P., Brodsky, I. E., Rahner, C., Woo, D. K., Erdjument-Bromage, H., Tempst, P., et al. (2011). TLR signalling augments macrophage bactericidal activity through mitochondrial ROS. Nature 472, 476-480. doi: 10.1038/ nature09973

Wilson, B. C., and Patterson, M. S. (2008). The physics, biophysics and technology of photodynamic therapy. Phys. Med. Biol. 53, R61-R109. doi: 10.1088/00319155/53/9/r01

Winter, S. E., Thiennimitr, P., Winter, M. G., Butler, B. P., Huseby, D. L., Crawford, R. W., et al. (2010). Gut inflammation provides a respiratory electron acceptor for Salmonella. Nature 467, 426-429. doi: 10.1038/nature 09415 
Wong, J., Chen, Y., and Gan, Y. H. (2015). Host cytosolic glutathione sensing by a membrane histidine kinase activates the type VI secretion system in an intracellular bacterium. Cell Host Microbe 18, 38-48. doi: 10.1016/j.chom.2015. 06.002

Wu, Y., Vulic, M., Keren, I., and Lewis, K. (2012). Role of oxidative stress in persister tolerance. Antimicrob. Agents Chemother. 56, 4922-4926. doi: 10. 1128/aac.00921-12

Yang, J. H., Wright, S. N., Hamblin, M., McCloskey, D., Alcantar, M. A., Schrubbers, L., et al. (2019). A white-box machine learning approach for revealing antibiotic mechanisms of action. Cell 177, 1649-1661.e9. doi: 10.1016/ j.cell.2019.04.016

Zhao, X., and Drlica, K. (2014). Reactive oxygen species and the bacterial response to lethal stress. Curr. Opin. Microbiol. 21, 1-6. doi: 10.1016/j.mib.2014. 06.008
Zhao, X., Hong, Y., and Drlica, K. (2015). Moving forward with reactive oxygen species involvement in antimicrobial lethality. J. Antimicrob. Chemother. 70, 639-642. doi: 10.1093/jac/dku463

Conflict of Interest: The authors declare that the research was conducted in the absence of any commercial or financial relationships that could be construed as a potential conflict of interest.

Copyright (c) $2021 \mathrm{Li}$, Zhou, Huang, Liao, Cheng and Ren. This is an open-access article distributed under the terms of the Creative Commons Attribution License (CC BY). The use, distribution or reproduction in other forums is permitted, provided the original author(s) and the copyright owner(s) are credited and that the original publication in this journal is cited, in accordance with accepted academic practice. No use, distribution or reproduction is permitted which does not comply with these terms. 